\title{
Approach to Hand Weakness from Clinical, Neurophysiological, Neuroradiological and Laboratory Data
}

\author{
Asmaa Belal ${ }^{1}$, Osama Ragab ${ }^{1 *}$, Heba Rafaat ${ }^{2}$, Ehab Shawky ${ }^{1}$, Tarek El-Gammall ${ }^{1}$ and Wael Fadel ${ }^{1}$ \\ ${ }^{1}$ Department of Neurology, Tanta University, Egypt \\ ${ }^{2}$ Department of Neurology, Cairo University, Egypt
}

*Corresponding author: Osama Ragab, Lecturer of Neurology, Tanta University, Egypt.

Received Date: January 08, 2020

Published Date: January 21, 2020

\section{Abstract}

Introduction: The current study aimed to develop an easy approach to the different neuromuscular disorders causing hand weakness.

Methods: 81 patients suffering from unilateral or bilateral hand weakness were subjected to neurological examination and electrophysiological assessment and imaging of the cervical cord by Magnetic resonance image for patients with mixed upper and lower motor neuron lesion.

Results: Inflammatory disorders were [24]; demyelinating neuropathy (5), axonal neuropathy (17), polymyositis (1) and myasthenic myopathy (1). Compressive disorders were [22]; median entrapment (11), radial and ulnar entrapment (5), degenerative radiculopathy, myelopathy (3), cervical syringomyelia (1) and thoracic outlet syndrome (1). Motor neuron disease (17). Hereditary disorders were [12]; myotonia (7), myopathy (2) and hereditary neuropathy (3). Traumatic disorders were [6]; radial and ulnar injury (4) and brachial plexus injury (2).

Discussion: We concluded that; inflammatory disorders were the most frequent causes of hand weakness. An algorithmic approach of hand weakness was concluded.

Keywords: Hand weakness; Neurophysiology; Neuropathy; Myopathy; Myasthenia; Diagnostic approach

\section{Introduction}

Distal pattern of weakness is a common clinical problem we usually meet in our clinical practice that needs workup to reach an accurate diagnosis [1]. Anatomically, disorders with hand weakness can be classified into central or peripheral neurologic disorders. Central disorders can result from lesions in the brain or spinal cord. Peripheral neurologic etiologies can be caused by lesions involving the different stations of lower motor neuron that start from the anterior horn cell to the muscle [2, 3]. According to the onset, hand weakness may be acute, subacute or chronic. Acute bilateral hand weakness and wasting represent a poorly recognized consequence of partial anterior spinal artery territory infarction [4]. Subacute hand weakness occurs as in brachial neuritis (BN) and mononeuritis multiplex. Chronic distal upper limb weakness may be due to peripheral nerve diseases as entrapment neuropathies, chronic inflammatory demyelinating polyneuropathy (CIDP), muscle diseases as distal myopathies, anterior horn cell disease and neuromuscular junction disorders as distal myasthenia gravis [5].

Electrophysiological studies are an extension of a clinical neurological examination as they help to characterize and localize the lesion through providing physiologic evidence of nerve dysfunction, differentiate a myopathic from a neurogenic disorder and detect anterior horn cell involvement. Therefore, they should be done in case when a lower motor neuron disorder is suspected [6]. Thus, historical, physical and electrodiagnostic information in addition to imaging findings are combined to confirm the most likely diagnosis and to guide future treatment [7].

\section{Methods}

This study was observational cross sectional study, conducted in Neuropsychiatry Department and the Centre of Neuropsychiatry 
and Neurosurgery, Tanta University Hospital. The study included 81 patients, 53 males and 28 females, with age ranged from 8 to 75 years; they all suffered from distal weakness of upper limbs. The studied patients were categorized into two main groups; group A included patients with unilateral weakness that was subdivided into group A1 that involved 20 patients with unilateral weakness of lower motor neuron lesion and group A2 that involved 4 patients with unilateral weakness of mixed upper and lower motor neuron lesion, and group B included patients with bilateral weakness that was subdivided into group B1 that had 40 patients with bilateral weakness of lower motor neuron lesion and group B2 that had 17 patients with bilateral weakness of mixed upper and lower motor neuron lesion. Patients with weakness of pure cerebral manifestations were excluded from the study.

All patients were subjected to detailed medical history including demographic data and analysis of symptoms including the onset, course and the duration of illness. The pattern, symmetry of the weakness and associated manifestations including sensory, autonomic or systemic were analyzed. Also past medical and family histories were taken. Full neurological examination according to the standard structural sheet of Neuropsychiatry Department was done for all patients. Motor nerve conduction studies and $\mathrm{F}$ wave of median, ulnar, peroneal and tibial nerves as well as sensory nerve conduction studies of median, ulnar and sural nerves were done for all patients. Repetitive nerve stimulation of the right ulnar and left facial nerves was performed for patients who were suspected to have myoneural junction defects. Needle EMG was conducted for all patients in the proximal and distal muscles of the cervical and lumbosacral segments and in the cranial muscles including the masseter, orbicularis oculi and orbicularis oris muscles, as well as in the dorsal paraspinals. Routine nerve conduction studies and EMG were done by conventional methods using Nicolet Biomedical VQ: VIASYS Healthcare. Inc, Madison Wisconsin 53711(Made in the USA-2007). The values of NCSs and EMG for each patient were compared with the average reference values of our laboratory.

Magnetic resonance image (MRI) of the cervical cord was done for patients with weakness of mixed upper and lower motor neuron lesion to assess the cervical cord and for patients with weakness of focal signs of lower motor neuron lesion suggesting for proximal pathology. Routine lab investigations were done for all patients including $\mathrm{CBC}$, kidney function (urea, creatinine), serum electrolytes ( $\mathrm{Na}, \mathrm{K}, \mathrm{Ca}$ ) and liver function. Further investigations were performed for selected patients when indicated as ESR, CRP, CPK, virology (HCV, HBV, HIV), immunological (Cryoglobulin, Ach-R antibodies, RF), hormonal (thyroid function, fasting and postprandial blood sugar), CSF analysis for (proteins, cells) and bone marrow aspiration. An Informed written consent for participation was obtained from all patients after full explanation of the procedure. The study protocol was approved by the Research Ethical Committee, Faculty of Medicine, Tanta University.

\section{Results}

Demographic data of the studied groups showed that most patients of the four groups were males in the same age group from rural areas with manual occupation and negative family histories as shown in Table 1.

Table 1: Demographic data of the studied groups.

\begin{tabular}{|c|c|c|c|c|c|}
\hline & & \multicolumn{2}{|c|}{ Unilateral group (A) $(n=24)$} & \multicolumn{2}{|c|}{ Bilateral group (B) $(n=57)$} \\
\hline & & LMN (A1) $(n=20)$ & Mixed (A2) $(n=4)$ & LMN (B1) $(\mathrm{n}=40)$ & Mixed (B2) $(n=17)$ \\
\hline \multicolumn{2}{|c|}{ Age (Mean \pm SD) } & $44 \pm 17.52$ & $45 \pm 13.43$ & $46.9 \pm 25.80$ & $43 \pm 22.67$ \\
\hline \multirow{2}{*}{ Sex } & Male & $14(70 \%)$ & $4(100 \%)$ & $22(55 \%)$ & 13 (76.5\%) \\
\hline & Female & $6(30 \%)$ & 0 & $18(45 \%)$ & $4(23.2 \%)$ \\
\hline \multirow{2}{*}{ Occupation } & Manual & $17(85 \%)$ & $3(75 \%)$ & $29(72.5 \%)$ & $13(76.5 \%)$ \\
\hline & Mental & $3(15 \%)$ & $1(25 \%)$ & $11(27.5 \%)$ & $4(23.2 \%)$ \\
\hline \multirow{2}{*}{ Residence } & Rural & $11(55 \%)$ & $1(25 \%)$ & $24(60 \%)$ & $12(70.6 \%)$ \\
\hline & Urban & $9(45 \%)$ & $3(75 \%)$ & $16(40 \%)$ & $5(29.4 \%)$ \\
\hline \multirow{2}{*}{$\mathrm{FH}$} & Positive & 0 & 0 & $8(20 \%)$ & 0 \\
\hline & Negative & $20(100 \%)$ & $4(100 \%)$ & $32(80 \%)$ & $17(100 \%)$ \\
\hline
\end{tabular}

LMN: Lower motor neuron; Mixed: Upper + lower motor neuron; FH: Family history; SD: Standard deviation; n: Number

The presenting symptoms were mainly weakness which was asymmetrical motor of gradual onset and slowly progressive course affecting both upper and lower limbs with wasting in most patients of the four groups with more affection of the cranial nerves in the bilateral groups as shown in Table 2 .

Table 2: Clinical data of the studied groups.

\begin{tabular}{|c|c|c|c|c|c|}
\hline \multicolumn{2}{|c|}{} & \multicolumn{2}{|c|}{ Unilateral group (A) (n=24) } & \multicolumn{2}{c|}{ Bilateral group (B) (n=57) } \\
\cline { 3 - 6 } & & LMN (A1) (n=20) & Mixed (A2) (n=4) & LMN (B1) (n=40) & Mixed (B2) (n=17) \\
\hline \multirow{2}{*}{ Onset } & Subacute & $11(55 \%)$ & $2(50 \%)$ & $8(20 \%)$ & $2(11.8 \%)$ \\
\cline { 2 - 6 } & Gradual & $9(45 \%)$ & $2(50 \%)$ & $32(80 \%)$ & $15(88.2 \%)$ \\
\hline
\end{tabular}




\begin{tabular}{|c|c|c|c|c|c|c|}
\hline \multirow{3}{*}{ Course } & & $\mathrm{P}$ & $9(45 \%)$ & $4(100 \%)$ & $37(92.5 \%)$ & $17(100 \%)$ \\
\hline & & RR & 0 & 0 & 0 & 0 \\
\hline & & $\mathrm{R}$ & $11(55 \%)$ & 0 & $3(7.5 \%)$ & 0 \\
\hline \multirow{2}{*}{$\begin{array}{c}\text { Main presenting } \\
\text { symptoms }\end{array}$} & \multicolumn{2}{|r|}{ Motor mainly } & $3(15 \%)$ & $4(100 \%)$ & $19(47.5 \%)$ & $12(70.6 \%)$ \\
\hline & \multicolumn{2}{|c|}{ Sensorimotor } & $17(85 \%)$ & 0 & $21(52.5 \%)$ & $5(29.4 \%)$ \\
\hline \multirow{4}{*}{ Weakness } & \multicolumn{2}{|r|}{ UL only } & 17 (85\%) & $3(75 \%)$ & $10(25 \%)$ & 0 \\
\hline & \multicolumn{2}{|r|}{$\mathrm{UL}+\mathrm{LL}$} & $3(15 \%)$ & $1(25 \%)$ & $30(75 \%)$ & $17(100 \%)$ \\
\hline & \multicolumn{2}{|r|}{ Symmetrical } & 0 & 0 & $16(40 \%)$ & 0 \\
\hline & \multicolumn{2}{|c|}{ Asymmetrical } & $20(100 \%)$ & $4(100 \%)$ & $24(60 \%)$ & $17(100 \%)$ \\
\hline \multirow{2}{*}{ Wasting } & \multicolumn{2}{|r|}{$+\mathrm{ve}$} & $15(75 \%)$ & $4(100 \%)$ & $33(82.5 \%)$ & $17(100 \%)$ \\
\hline & \multicolumn{2}{|r|}{-ve } & $5(25 \%)$ & 0 & $7(17.5 \%)$ & 0 \\
\hline \multirow{2}{*}{ Sensory } & \multicolumn{2}{|r|}{+ ve } & 17 (85\%) & 0 & $28(70 \%)$ & $5(29.4 \%)$ \\
\hline & \multicolumn{2}{|r|}{-ve } & $3(15 \%)$ & $4(100 \%)$ & $12(30 \%)$ & $12(70.6 \%)$ \\
\hline \multirow{5}{*}{ Reflexes } & \multirow{2}{*}{ Sup } & Normal & $20(100 \%)$ & $1(25 \%)$ & $40(100 \%)$ & 0 \\
\hline & & Abnormal & 0 & $3(75 \%)$ & 0 & $17(100 \%)$ \\
\hline & \multirow{3}{*}{ Deep } & Normal & $14(70 \%)$ & 0 & $15(37.5 \%)$ & $1(5.9 \%)$ \\
\hline & & Hypo/areflexia & $6(30 \%)$ & 0 & $24(60 \%)$ & $1(5.9 \%)$ \\
\hline & & Hyperreflexia & 0 & $4(100 \%)$ & $1(2.5 \%)$ & $15(88.2 \%)$ \\
\hline \multirow{2}{*}{ Cr. N. affection } & & $+\mathrm{ve}$ & 0 & $3(75 \%)$ & $9(22.5 \%)$ & $10(58.8 \%)$ \\
\hline & & -ve & $20(100 \%)$ & $1(25 \%)$ & $31(77.5 \%)$ & $7(41.2 \%)$ \\
\hline \multirow{2}{*}{ Weakness } & & Motor & $6(30 \%)$ & $4(100 \%)$ & $12(30 \%)$ & $12(70.6 \%)$ \\
\hline & \multicolumn{2}{|c|}{ Sensorimotor } & $14(70 \%)$ & 0 & $28(70 \%)$ & $5(29.4 \%)$ \\
\hline
\end{tabular}

LMN: Lower motor neuron; Mixed: Upper + lower motor neuron; P: Progressive; RR: Remitting-relapsing; R: Regressive; Sup: Superficial; Cr. N: Cranial nerve; +ve: Positive; -ve: Negative; UL: Upper limb; LL: Lower limb; n: Number

Electrophysiological study showed that the anterior horn cell peripheral nerve diseases (root, plexus and nerve) either axonal or lesion was found mainly in the bilateral mixed group. Muscle and myoneural diseases were found in the bilateral LMN group while demyelinating were found in the LMN groups either unilateral or bilateral as shown in Table 3.

Table 3: Neurophysiological data of the studied groups.

\begin{tabular}{|c|c|c|c|c|c|c|}
\hline & \multicolumn{2}{|c|}{ Unilateral group $(A)(n=24)$} & \multicolumn{2}{|c|}{ Bilateral group (B) $(n=57)$} \\
\hline & & & $\operatorname{LMN}(\mathrm{A} 1)(\mathrm{n}=20)$ & Mixed (A2) $(n=4)$ & LMN (B1) $(n=40)$ & Mixed $(B 2(n=17)$ \\
\hline \multirow{10}{*}{ EDXs } & & Neuronopathy & $1(5 \%)$ & $4(100 \%)$ & 0 & $13(76.5 \%)$ \\
\hline & & Axonopathy (A) & $9(45 \%)$ & 0 & $19(36.8 \%)$ & $4(23.5 \%)$ \\
\hline & & relinopathy (Dem) & $5(25 \%)$ & 0 & $8(26.3 \%)$ & 0 \\
\hline & & Mixed (A+Dem) & $5(25 \%)$ & 0 & $7(36.8 \%)$ & 0 \\
\hline & & Normal NCSs & 0 & 0 & $6(15 \%)$ & 0 \\
\hline & \multirow{4}{*}{ EMG } & Neuropathic & $20(100 \%)$ & $4(100 \%)$ & $29(72.5 \%)$ & $17(100 \%)$ \\
\hline & & Myopathic & 0 & 0 & $8(20 \%)$ & 0 \\
\hline & & Normal (myotonic & \multirow{2}{*}{0} & \multirow{2}{*}{0} & \multirow{2}{*}{$3(7.5 \%)$} & \multirow{2}{*}{0} \\
\hline & & discharges) & & & & \\
\hline & \multicolumn{2}{|r|}{ Myoneural } & 0 & 0 & $1(2.5 \%)$ & 0 \\
\hline
\end{tabular}

LMN: Lower motor neuron; Mixed: Upper + lower motor neuron; EDSs: Electrodiagnostic studies; NCSs: Nerve conduction studies; EMG: Electromyography; n: Number; EDX: Electrodiagnostic studies

Radiological data of the studied groups showed that remarkable changes in the cervical MRI either disc or cord pathology were found in few patients in the bilateral mixed group, and unremarkable changes in disc or spondylosis in few patients of the remaining groups as shown in Table 4.
From the clinical, neurophysiological and neuroradiological findings of the patients, they were categorized into 5 groups: inflammatory which was the more frequent followed by compressive, degenerative, hereditary and finally the traumatic group as shown in Table 5. 
Table 4: Radiological data of the studied groups.

\begin{tabular}{|c|c|c|c|c|c|c|}
\hline & & & \multicolumn{2}{|c|}{$\begin{array}{l}\text { Unilateral group }(A) \\
\qquad(n=24)\end{array}$} & \multicolumn{2}{|c|}{$\begin{array}{c}\text { Bilateral group (B) } \\
(n=57)\end{array}$} \\
\hline & & & LMN (A1) $(n=20)$ & Mixed (A2)(n=4) & LMN $(B 1)(n=40)$ & Mixed (B2) $(n=17)$ \\
\hline \multirow{8}{*}{$\begin{array}{c}\text { Cervical } \\
\text { MRI }\end{array}$} & \multirow{4}{*}{ Disc } & UCD & $1(5 \%)$ & 0 & 0 & $1(5.9 \%)$ \\
\hline & & LCD & $2(10 \%)$ & 0 & 0 & $2(11.8 \%)$ \\
\hline & & Remarkable & $1(5 \%)$ & 0 & 0 & $2(11.8 \%)$ \\
\hline & & Unremarkable & $1(5 \%)$ & 0 & 0 & 0 \\
\hline & \multirow{2}{*}{ Spondylosis } & Diffuse & $1(5 \%)$ & 0 & 0 & $2(11.8 \%)$ \\
\hline & & Focal & 0 & $2(50 \%)$ & $3(7.5 \%)$ & 0 \\
\hline & \multirow{2}{*}{ Cervical cord lesion } & $+\mathrm{ve}$ & 0 & 0 & 0 & $3(17.6 \%)$ \\
\hline & & -ve & $20(100 \%)$ & $4(100 \%)$ & $40(100 \%)$ & $14(82.4 \%)$ \\
\hline
\end{tabular}

LMN: Lower motor neuron; Mixed: Upper + lower motor neuron; MRI: Magnetic Resonance Image; UCD: Upper cervical disc; LCD: Lower cervical disc; +ve: Positive; -ve: Negative; n: Number

Table 5: The frequency of neuromuscular disorders in the groups.

\begin{tabular}{|c|c|c|c|c|}
\hline & Etiology & Disorders (=81 patients) & No & Total No.(\%) \\
\hline \multirow{7}{*}{ 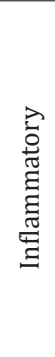 } & \multirow[b]{2}{*}{ Demyelinating } & Chronic inflammatory demyelinating polyneuroradiculopathy & 3 & \multirow{7}{*}{$\begin{array}{c}(24) \\
(29.6 \%)\end{array}$} \\
\hline & & Multifocal motor neuropathy with conduction block & 2 & \\
\hline & \multirow{5}{*}{ Non Demyelinating } & Sensorimotor axonal polyneuroradiculopathy & 9 & \\
\hline & & Sensorimotor axonal polyneuropathy & 4 & \\
\hline & & Mononeuritis multiplex & 4 & \\
\hline & & Chronic polymyositis & 1 & \\
\hline & & Myasthenic myopathy & 1 & \\
\hline & \multirow{9}{*}{ Compressive } & Carpal tunnel syndrome & 10 & \multirow{9}{*}{$\begin{array}{c}(22) \\
(27.2 \%)\end{array}$} \\
\hline & & Radial nerve entrapment at the spiral groove & 3 & \\
\hline & & Ulnar nerve entrapment at the elbow & 2 & \\
\hline & & Pronator teres syndrome & 1 & \\
\hline & & Thoracic outlet syndrome & 1 & \\
\hline & & $\begin{array}{l}\text { Left partial axonal lesion affecting the brachial plexus at cords level } \\
\text { (Panplexopathy) }\end{array}$ & 1 & \\
\hline & & $\mathrm{C} 8, \mathrm{~T} 1$ radiculopathy & 1 & \\
\hline & & Cervical Syringomyelia & 1 & \\
\hline & & $\begin{array}{l}\text { Lower cervical myelopathy (Roots in both ULs with spastic para- } \\
\text { paresis) due to C5,6,7 disc prolapsed } \\
\text { Polyradiculopathy of both ULs due to advanced spondylodegener- } \\
\text { ative changes of the cervical spine with spastic paraparesis }\end{array}$ & $\begin{array}{l}1 \\
1\end{array}$ & \\
\hline & Degenerative & Motor neuron disease & 17 & $\begin{array}{c}17)) \\
(20.9 \%)\end{array}$ \\
\hline & \multirow{5}{*}{ Hereditary } & Myotonia congenita & 4 & \multirow{5}{*}{$\begin{array}{c}(12) \\
(14.8 \%)\end{array}$} \\
\hline & & Myotonia dystrophica & 3 & \\
\hline & & Hereditary sensorimotor polyneuropathy & 3 & \\
\hline & & Inclusion body myopathy & 1 & \\
\hline & & Distal myopathy & 1 & \\
\hline & \multirow{3}{*}{ Traumatic } & Radial nerve injury & 2 & \multirow{3}{*}{$\begin{array}{c}(6) \\
(7.4 \%)\end{array}$} \\
\hline & & Ulnar nerve injury & 2 & \\
\hline & & Chronic partial axonal lesion of the right brachial plexus & 2 & \\
\hline
\end{tabular}

C: Cervical; T: Thoracic; UL: Upper limb

\section{Discussion}

The studied patients with hand weakness were 81. 30 patients had motor weakness and 51 had sensorimotor weakness. Patients with motor weakness were motor neuron disease (MND), multifocal motor neuropathy (MMNCB), myotonic disorders including myotonia congenita (MC) and dystrophic myotonia (DM), polymyositis, distal myopathy and myasthenic myopathy. In the MND and MMNCB patients, the clinical presentation was similar 
to some extent but with some differences as follows: the weakness and wasting in MMNCB were mainly distal within specific nerve territory with affection of lower limbs in the same manner in absence of upper motor neuron lesion (UMNL) signs while weakness and wasting in MND were severe and diffuse affecting the distal and proximal muscles with lower limb affection, upper motor neuron lesion (UMNL) signs and bulbar manifestations (except in the monomelic patient). This agreed with [8,9] who reported that MMN is a pure motor neuropathy characterized by slowly progressive, asymmetrical and distal weakness which predominantly affects ULs. It also matched with $[10,11]$ who showed that ALS is characterized by mixed upper and lower MNL signs which can lead to asymmetric distal weakness and wasting that usually begin focally in the limbs in two third of patients without sensory loss and then progress to contagious regions of the body.

Electrophysiologically, normal sensory nerve conduction studies (SNCSs) with motor axonopathy and reduced compound muscle action potentials (CMAP) were similar to some extent but with some differences that motor axonopathy were severe and diffuse in MND with negative inching for conduction block (CB). Denervation potentials were more profuse in MND and mild in MMNCB with a neurogenic pattern motor unit action potentials (MUPs) that were related to nerve territory in MMNCB and diffuse in MND.

Two forms of myotonia were found (MC and DM). Some clinical clues were found and they helped in differentiating both of them as characteristic facies, distal and proximal muscle weakness and wasting, ophthalmoparesis and systemic manifesations as cataract in patient with DM. Electrophysiologically, they were similar to some extent (normal NCSs apart from mild motor axonopathy in some patient with DM and myotonic potentials in EMG) but the pattern of recruitment and MUPs were differentiating since myopathic pattern was found in DM, and normal MUPs with average recruitment were found in most patients with MC and this matched with $[12,13]$ who reported that MC usually presents with mild, progressive, distal weakness that induced by movement after rest and relieved by repetitive movement known as "the warm-up effect" and doesn't cause muscle wasting.

Regarding DM, this agreed with [14-15] who reported that it begins with progressive muscle weakness, stiffness and wasting involving the legs, hands, neck and face with other signs and symptoms as cataracts, type- 2 diabetes, cardiac conduction defects and hormonal changes that may cause balding or infertility. In the patient with chronic polymyositis, the clinical picture was similar to MND but the history was differentiating from myalgia at the onset; symmetrical proximal weakness started in lower then upper limbs, and neck muscle became affected with late affection of distal muscles. Electrophysiologically, the patient showed only motor axonopathy and denervation potentials as MND but with myopathic pattern. The diagnosis was confirmed by markedly elevated ESR and CPK and this was compatible with [16-17] who noted that polymyositis commonly presents with proximal and often symmetrical weakness over weeks to months. Patients showed both myopathic and neuropathic features in EMG and serum CK is always essentially elevated.

The last type of muscle disease in this study was the myopathy with distal muscle weakness (Inclusion body myopathy (IBM) and distal myopathy). The clinical picture was confusing since the distal type of weakness is usually specific for neuropathy not myopathy. But the clinical clues were gradually asymmetrical onset of IBM in the proximal LLs muscles which is specific for muscle disease followed by hand affection without any sensory disturbances. This agreed with [18] who reported that IBM causes progressive asymmetrical muscle weakness and wasting involving distal forearm muscles (wrist, finger flexors) and proximal leg (quadriceps) with sparing of facial muscles.

In distal myopathy, the clinical clues were gradual symmetrical hand weakness with characteristic fingers drop (4th, 5th) with distal and proximal LLs weakness and wasting and this matched with [19] who described Welander distal myopathy (Provisional diagnosis of the patient) as late-onset weakness of the finger extensors followed by intrinsic hand and distal LL muscles. As the myopathy progresses, wasting develops and proximal muscles may become involved. Electrophysiological findings that weighted the diagnosis of IBM and welander myopathy were normal NCSs in both types apart from mild motor axonopathy and presence of denervation potentials with mixed myopathic and neurogenic pattern in the affected muscles in IBM and myopathic pattern in distal myopathy and mildly elevated CPK in both types. This agreed with [20] who reported in their study on 3 Saudi families with HIBM that CK was mildly elevated with normal NCSs and EMG was consistent with primary myopathy.

The last cause of motor weakness was myasthenic myopathy in which the history of fatigable symmetrical proximal weakness was present. Hand affection was a part of generalized weakness and wasting that developed with long standing disease and this matched with [21] who reported that symptoms of myasthenia gravis pass through phases; fluctuation phase followed by active phase then inactive phase and finally the burnt-out phase that occurs after 1520 years in which the weakness often becomes fixed and the most severely involved muscles are frequently atrophic.

Patients with sensorimotor weakness were median nerve entrapment (carpal tunnel syndrome (CTS) and pronator syndrome (PS)), radial nerve lesion (entrapment at the spiral groove (SG) and injury), ulnar nerve lesion (entrapment at the elbow (UNEE) and injury), brachial plexus lesion, cervical radiculopathy, myelopathy and syringomyelia, mononeuritis multiplex (MM), thoracic outlet syndrome (TOS), chronic inflammatory demyelinating polyneuroapthy (CIDP), multifocal acquired demyelinating sensory and motor polyneuropathy (MADSAM), hereditary neuropathy (HN), sensorimotor axonal neuropathy and polyradiculopathy.

Clinically, CTS and PS were confusing but paresthesia distribution was differentiating since it was found in the lateral 
three and half fingers only in CTS while, in PS, the lateral two third of the palm was also included and this matched with [22-24] who showed that CTS and PS may be easily mistaken but the sensory loss in both of them is variable. The differentiation between both became complete electrophysiologically since CTS showed delayed sensorimotor distal latencies (DL) or sensorimotor axonopathy (reduced CMAP and SNAP) of the median nerve with chronic neurogenic pattern in the abductor pollicis brevis (APB) muscle only while the conduction was normal and neurogenic pattern was found in APB, flexor pollicis longus (FPL) and flexor digitorum profundus (FDP) (median half) in PS.

Also, UNEE, TOS and C8 radiculopathy were clinically confusing, but also paresthesia distribution was differentiating since it was found in the medial one and half fingers and medial third of the hand in UNEE while in the medial aspect of arm, forearm, hand and little finger (radicular) in C8 radiculopathy and in the medial aspect of the forearm in TOS, and this matched with [25-27] who reported that in UNEE, there is dysesthesias in the 4th and 5th fingers with proximal medial elbow pain, followed by progressive weakness and wasting of intrinsic muscles with ulnar claw posture. Regarding the TOS, this was compatible with [28] who clarified that paresthesia is observed in all 5 fingers but more in the 4 th and 5 th digits as well as medial forearm with global hand weakness and wasting especially in hypothenar and lateral thenar muscles.

Electrophysiologically, the differentiation among them became complete since CB was found at the elbow in UNEE with the absence of its SNAP, and neurogenic pattern was found in the first dorsal interosseus (FDI), abductor digiti minimi (ADM) and flexor carpi ulnaris (FCU) while in C8 radiculopathy, NCSs were normal, and neurogenic pattern was found in APB, FDI and EIP. On the other hand, in TOS, SNAPs of ulnar and medial antebrachial nerves were low in relative to healthy side with low CMAP of median nerve, and neurogenic pattern was more in APB and less in FDI and EIP. The diagnosis was confirmed by the imaging of cervical spine that showed disc bulge in radiculopathy and cervical rib in TOS. This agreed with [29] who highlighted that the most reliable finding in UNEE is CB across the elbow and it also agreed with [30] who reported that, in the cervical radiculopathy, the SNCs are normal as the lesion is preganglionic and MNCs are normal unless there is a severe axon loss with chronic neurogenic pattern in a clear myotomal distribution. Regarding the TOS, this was confirmed by [31] who mentioned that low CMAP of median nerve with its normal SNAP amplitude, low ulnar and medial antebrachial SNAPs with neurogenic pattern in median to be more than ulnar-innervated C8TI muscles were found. Cervical MRI showed moderate C7-T1 disc prolapse in cervical radiculopathy with cervical rib in TOS patient. This was the same given by $[32,33]$ who reported that, in younger patients, disc trauma and herniation are the most frequent causes of impingement and bone anomalies as cervical rib is frequent in TOS patients.

Wrist and fingers drop was the presenting symptom in radial entrapment at SG and in brachial panplexopathy. But the differentiating points were latent in the fact that wrist and fingers drop and mild paresthesia in the back of hand and forearm were the only findings in radial nerve entrapment at the SG. This corresponded with [34] who showed that radial lesion at the arm includes weakness in the brachioradialis (BR), supinator, extensor carpi radialis longus and brevis (ECRL, B) with sensory loss in the posterior forearm, dorsum of the hand and lateral three and half fingers, and it also agreed with [35] who reported that brachial plexus injuries usually affect younger males because of their association with trauma.

Electrophysiologically, the differentiation was completed since, in radial entrapment, $\mathrm{CB}$ at the SG with normal SNAP and reduced recruitment in extensor indicis proprius (EIP), extensor digitorum communis (EDC), ECR and BR were found while in brachial panplexopathy at cords level, SNAPs recorded from all fingers were absent with low CMAPs of ulnar, median and radial nerves. This matched with [36] who explained in his study that approximately $60 \%$ of patients with radial neuropathy at the SG showed CB at the SG. This result also met that by [37] who reported that patients with C8- T1 (lower trunk) lesion showed absent SNAPs from little finger with neurogenic pattern in EDC, EIP, FPL, lumbricals, interossei, thenar and hypothenars while in C5- T1 (panplexopathy) lesion, SNAPs from all fingers were absent with neurogenic pattern in proximal and distal muscles.

In traumatic nerve injury, we had radial and ulnar lesions. The clues were the history of trauma, the site of injury that was suggestive for the injured nerves and the clinical presentation, as lesion in back of arm led to radial injury with resulting wrist and fingers drop, and lesion in the medial back of elbow and medial forearm led to ulnar injury with resulting partial ulnar claw hand and wasting in hypothenar muscles. Electrophysiologically, there were mixed sensorimotor axonopathy with neurogenic patten in the muscles distal to the site of injury.

This study included patients with mixed upper and lower MNL signs whose cervical MRI showed focal cord lesion as degenerative lower cervical myelopathy and cervical syringomyelia. In myelopathy, the clinical clues were old age of patients, gradual onset of radicular paresthesia, weakness and wasting, and late spastic paraparesis with sphincteric disturbances. Electrophysiologically, normal conductions with chronic neurogenic pattern in the lower cervical roots (C7.8 and T1) muscles and degenerative lower cervical myelopathy in the cervical MRI were found and this matched with [30-38] who reported that cervical spondolytic myelopathy is the commonest spinal cord disorder in old people with a slow insidious onset of radicular paresthesia followed by fine motor skills dysfunction and atrophy of the hand or shoulder.

In cervical syringomyelia, the clues were gradual onset of jacket paresthesia, proximal and distal upper limbs weakness and wasting in asymmetrical manner with later spastic paraparesis, normal conductions with chronic neurogenic pattern in the distal and proximal muscles of upper limbs and long syrinx extending from C2- T1 with Arnold Chiari malformation in cervical MRI. This 
agreed with $[39,40]$ who reported that symptoms in syringomyelia develop slowly with dissociative paresthesias in a cape or half-cape distribution, and atrophy of intrinsic hand muscles or fasciculations may also be an early finding; signs of myelopathy in lower limbs can become evident as the disease progresses.

This study included another demyelinating neuropathy (CIDP) like MMN but with sensorimotor presentation. Such manifestations of CIDP can be easily differentiated from each other clinically and electrophysiologically. Clinically, CIDP had a subacute onset, ascending nearly symmetrical weakness (proximal and distal) and wasting with glove and stoke paresthesia and observable myokymic movements in the proximal muscles in one patient. This matched with [41-42] who described that typical CIDP patients showed progression of proximal and distal muscle weakness over at least an 8-weeks period associated usually with paresthesia in a stocking-glove pattern.

The electrophysiological criteria were sensorimotor multifocal demyelinating features with secondary axonopathy and neurogenic pattern in the distal and proximal muscles, and this was compatible with who described the criteria of CIDP diagnostic criteria [43].

The MADSAM (phenotype of CIDP) was similar to MMNCB but with nerve paresthesia and asymmetrical mixed sensorimotor Multifocal demyelinating features. This matched with $[44,45]$ who reported that sensori-motor manifestations in MADSAM usually start in upper limbs but with chronic onset and symmetrical pattern, and NCSs can provide mixed results (demyelinating and axonal) because of secondary axonal degeneration within time. The last demyelinating neuropathy in this study was the hereditary neuropathy (CMT). The clinical clues were childhood or young age of onset, positive family history, bilateral symmetrical ascending distal weakness and wasting (inverted champagne bottle), mild glove and stoke paresthesia, skeletal deformities (pes cavus). Electrophysiological clues were diffuse symmetrical sensorimotor demyelinating features (no CB) with secondary axonopathy in two patients and primary sensorimotor axonopathy in one patient. This agreed with [46] who reported that CMT patients manifest symmetric, slowly progressive distal motor neuropathy of the arms and legs usually in the 1st to 3rd decade resulting in weakness and atrophy of the muscles in the feet and/or hands with mild distal sensory loss and pes cavus.

In mononeuritis multiplex, the diagnostic points were subacute sensorimotor manifestations in specific nerves with electrophysiological evidence of sensorimotor axonopathy in separate nerves in upper and lower limbs, and mild demyelinating features in one patient. MM is frequently presented in asymmetrical fashion with an acute or subacute onset of sensorimotor deficits, and its diagnosis was confirmed with asymmetrical severe sensorimotor axonal loss more in the upper limbs or by the presence of $\mathrm{CB}$, contrasting with a mild degree of demyelination outside the blocked nerve territory $[47,48,49]$.

Primary etiologies were found in some patients of MM as rheumatoid arthritis and psoriasis, and one of patients was poly substance abuse for four years; this neuropathy may be attributed to the abusing substances as it was reported by [50] that abuse of n-hexane (common in teenage boys) may cause a symmetrical sensorimotor axonal neuropathy which starts insidiously or subacutely with a symmetrical fashion in hands and feet with dysautonomia. Few patients had no identifiable etiology for their neuropathy and this matched with [48-51] who found that 33\% of cases originate from unidentifiable causes in which vasculitis may be the etiology; it was reported that asymmetry of neuropathy at the onset may be indicative of a multifocal process that quickly becomes confluent as in CIDP and vasculitis [52].

This study included diabetic sensori-motor axonal polyneuropathy. The findings revolved around long standing diabetes, old male patients, gradual onset of nearly symmetrical ascending distal sensorimotor weakness with electrophysiological evidence of symmetrical sensori-motor axonopathy and neurogenic pattern in distal muscles of upper and lower limbs and this agreed with [53] who reported that diabetic sensorimotor neuropathy represents a diffuse symmetrical length-dependent neuropathy with predominant sensorimotor axonopathy. Finally, polyneuroradiculopathy was found more in males in middle and old age groups with gradual onset in most patients. The clues were asymmetrical proximal (more) and distal weakness of upper and lower limbs, radicular and glove, stoke paresthesia, Cranial nerves affection (facial diplegia, ophthalmoparesis) and electrophysiological evidence of sensori-motor axonopathy with neurogenic pattern in the distal, proximal, paraspinals and facial muscles (frontalis, few patients), and this was compatible with [54] who reported that clinical manifestations of polyradiculopathy are variable but often include symmetric or asymmetric distal and proximal weakness with a variable degree of sensory loss and it also agreed with [13] who reported that electrophysiological findings of polyneuroradiculopathy involve sensorimotor axonopathy and normally or mildly delayed FL with denervation in paraspinals, distal and proximal muscles.

ESR was elevated in few patients; one of them was male with subacute onset of weakness and generalized lymphadenopathy, and he was diagnosed later to have lymphoma, another was female with gradual onset of the disease and others were lab free. CSF proteins were elevated in three patients; two of them were diabetic; one presented by subacute onset and another with gradual onset Of weakness mainly proximal and then diagnosed with diabeticCIDP since it was reported by [55] those patients with D-CIDP have a slow and usually monophasic course in which worsening occurs over months while diabetic polyneuropathy generally has a much slower course in which worsening occurs over many years. The 3rd one was known to have hereditary neuropathy, presented by subacute onset of increased weakness mainly proximal and showed initial response to steroids. The remaining patients were lab free.

From clinical, neurophysiological, neuroradiological and laboratory findings of the mentioned neuromuscular disorders, we had an algorithm for easy approach of hand weakness in which the patients were divided into patients with unilateral and bilateral 
weakness, motor and sensorimotor weakness, symmetrical and shown in Figure 1A, 1B, 1C ,1D. asymmetrical weakness with or without lower limb affection as

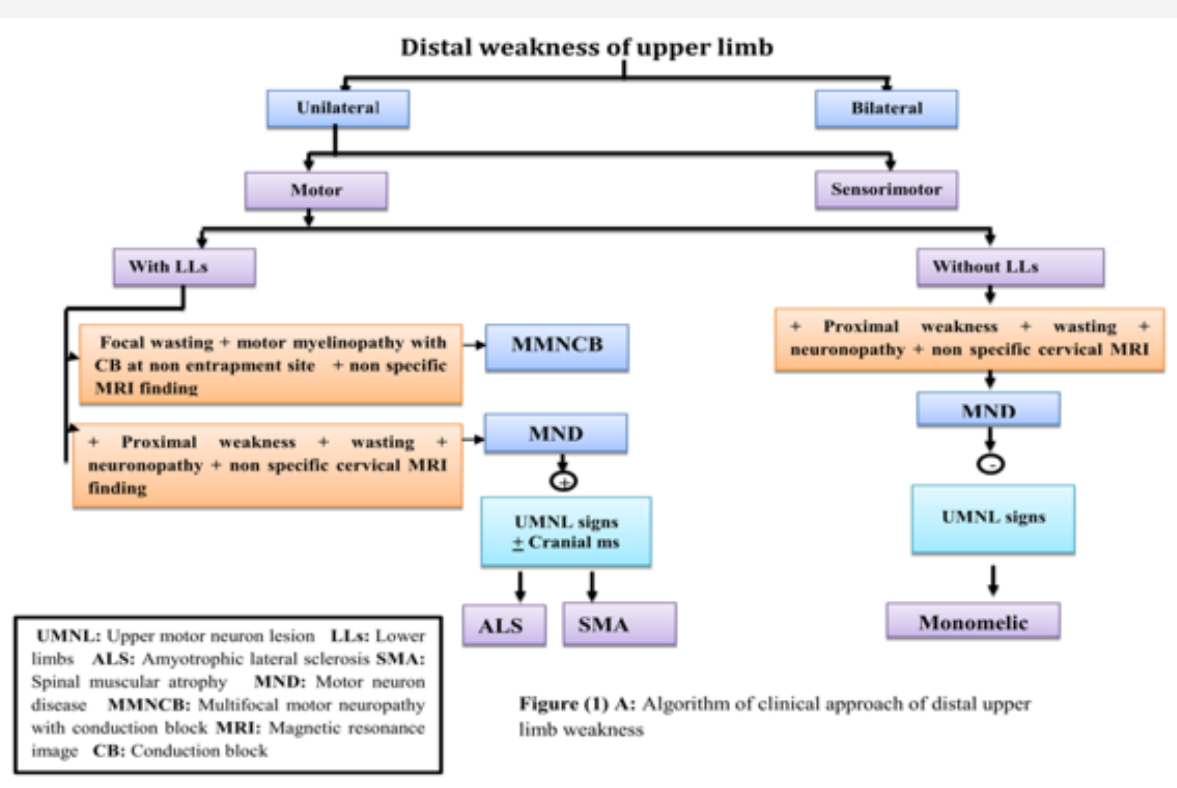

Figure 1(A): Algorithm of clinical approach of distal upper limb weakness.

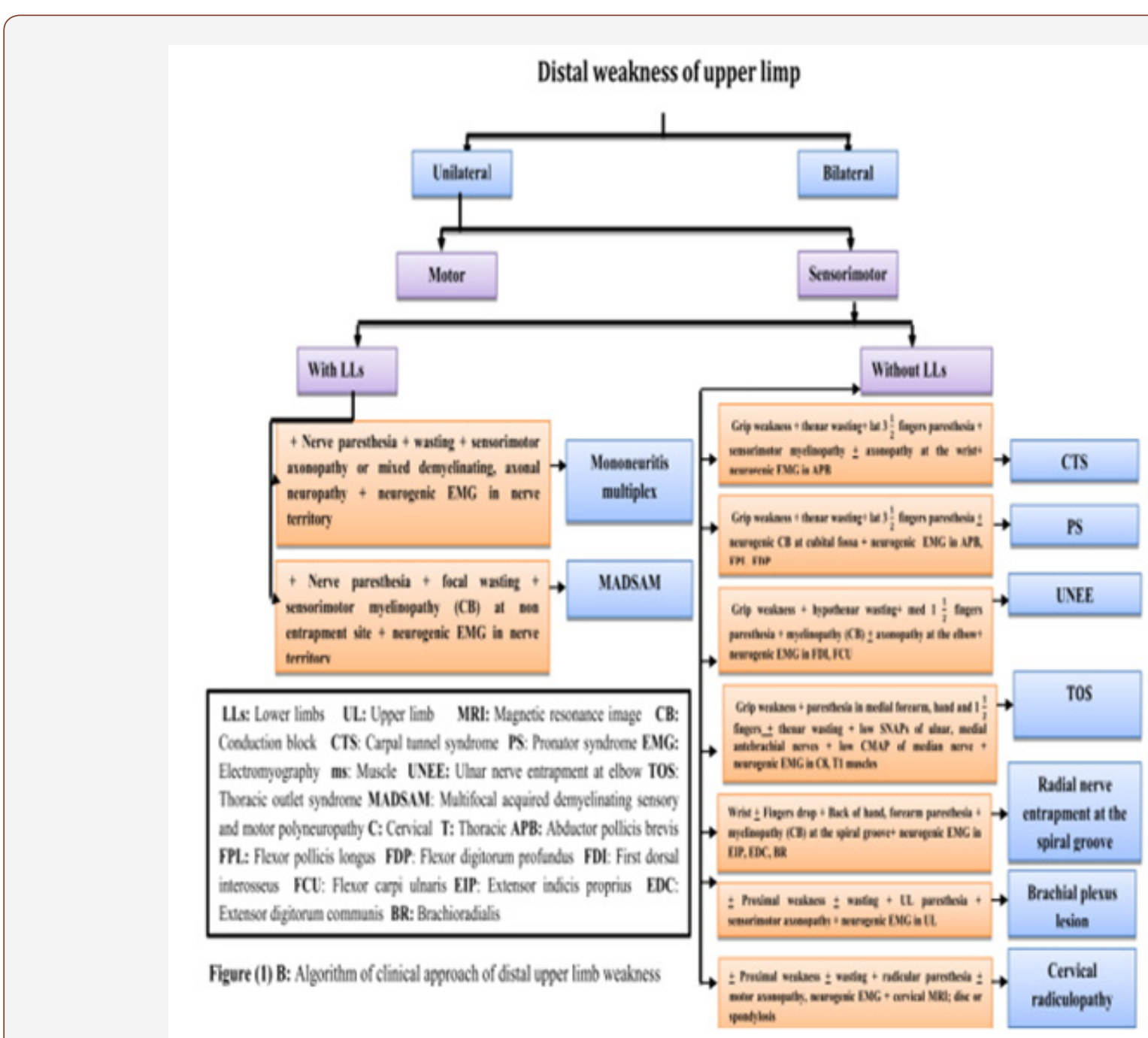

Figure 1(B): Algorithm of clinical approach of distal upper limb weakness. 


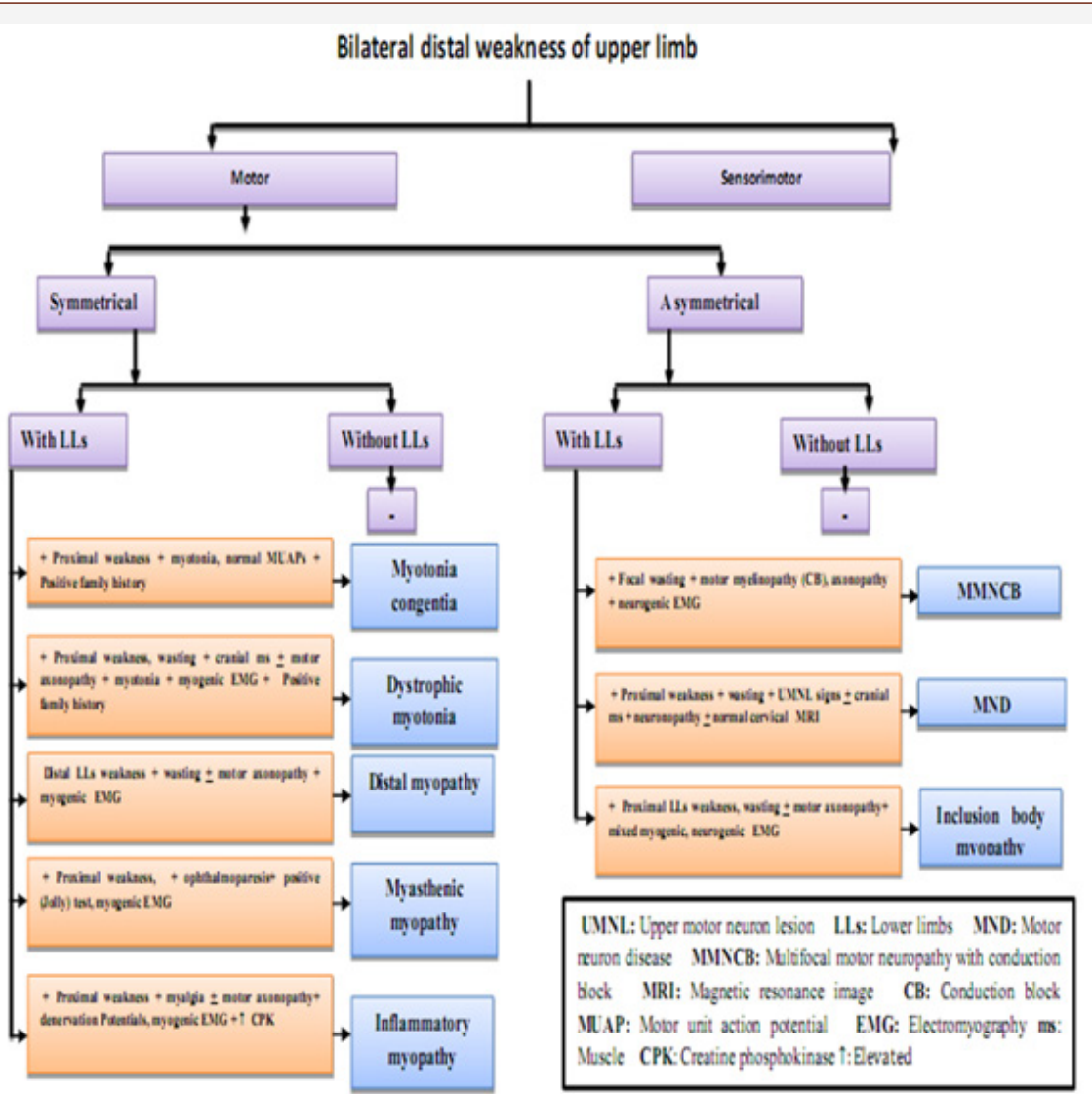

Figure 1(C): Algorithm of clinical approach of distal upper limb weakness.

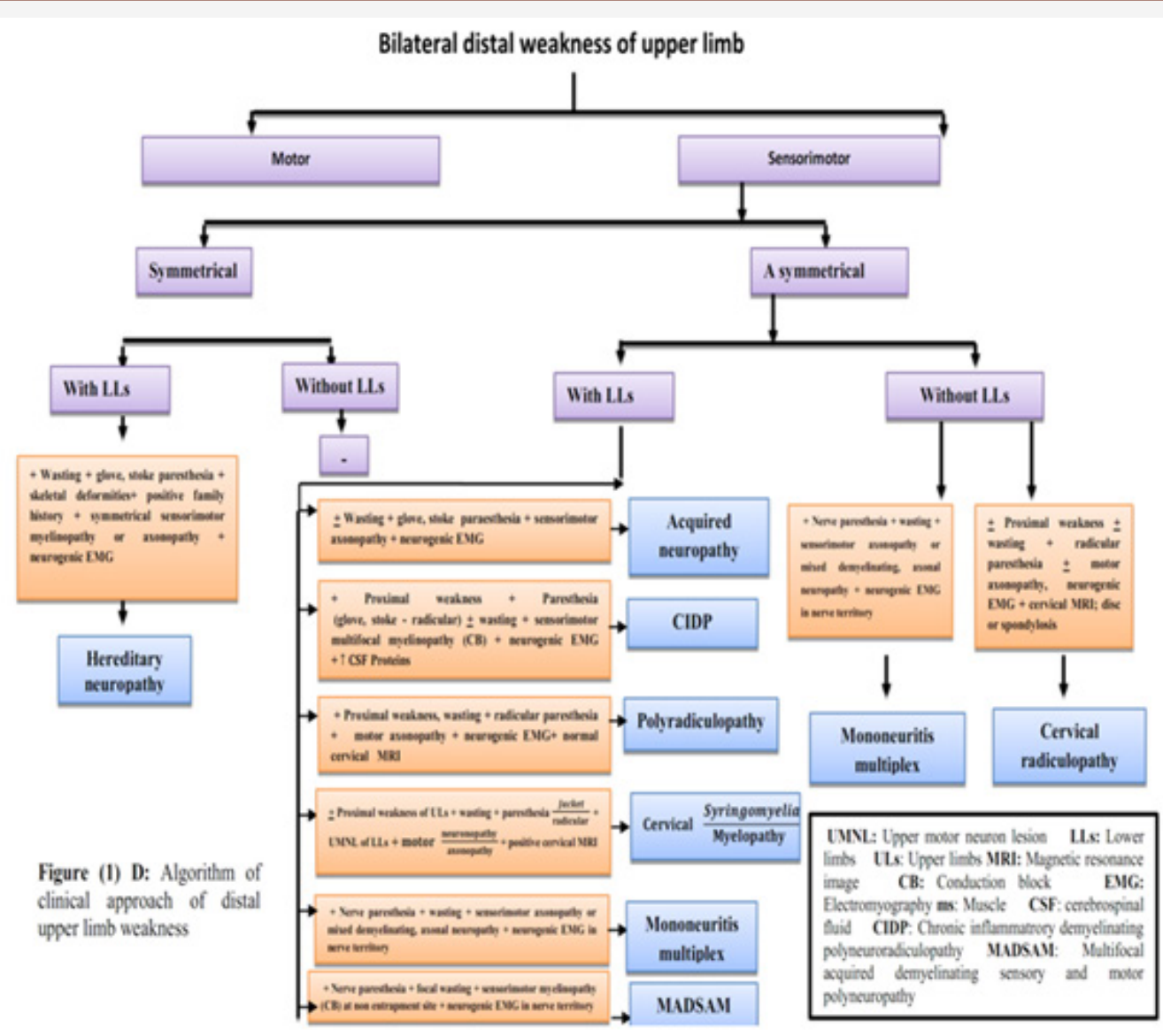

Figure 1(D): Algorithm of clinical approach of distal upper limb weakness. 


\section{Conclusion}

Inflammatory disorders were the most frequent causes of hand weakness followed by compressive, degenerative, hereditary and traumatic ones.

\section{Acknowledgement}

We wish to express our deep thanks to our patients and their families, also we should thank Mrs Hagar Abo El-fath Belal for her help in editing that manuscript.

\section{Conflict of Interest}

No conflict of interest.

\section{References}

1. Jackson CE (2008) A clinical approach to muscle diseases. In Semin Neurol 28(02): 228-240.

2. Chen PL, Hsu HY, Wang PY (2006) Isolated hand weakness in cortical infarctions. J Formos Med Assoc 105(10): 861-865.

3. Swash M, Schwartz MS (2013) Neuromuscular diseases. A practical approach to diagnosis and management. Springer Science \& Business Media: 14.

4. Balaratnam M, Pullicino P (2009) Teaching NeuroImages: Acute bilateral hand weakness from anterior spinal artery territory cord ischemia. Neurology 73(3): e13.

5. leeson E, Wolfson L (2010) Neuromuscular diseases in geriatric patients. Clin Geriatr 18: 17-24.

6. Mbuya SO (2006) The role of neuro-electrophysiological diagnostic tests in clinical medicine. East Afr Med J 83(1): 52-60.

7. Oryema J, Ashby P, Spiegel S (1990) Monomelic atrophy. Can J Neurol Sci 17(2): 124-130.

8. Matsui N (2012) Multifocal motor neuropathy: current review of epidemiology and treatment. Rinsho Shinkeigaku 52(11): 920-922.

9. Muley SA, Parry GJ (2012) Multifocal motor neuropathy. J Clin Neurosci 19(9): 1201-1209.

10. Williams TL (2013) Motor neurone disease: diagnostic pitfalls. Clin Med 13(1): 97-100.

11. Foster LA, Salajegheh MK (2019) Motor neuron disease: pathophysiology, diagnosis, and management. Am J Med 132(1): 32-37.

12. Portaro S, Cacciola A, Naro A, Milardi D, Morabito R, et al. (2018) A case report of recessive myotonia congenita and early onset cognitive impairment: Is it a causal or casual link?. Medicine 97(22): e10785.

13. Preston DC, Shapiro BE (2012) Electromyography and Neuromuscular Disorders E-Book: Clinical-Electrophysiologic Correlations (Expert Consult-Online and Print). Elsevier Health Sciences.

14. Thomas JD, Sznajder ŁJ, Bardhi O, Aslam FN, Anastasiadis ZP, et al. (2017) Disrupted prenatal RNA processing and myogenesis in congenital myotonic dystrophy. Genes Dev 31(11): 1122-1133.

15. Hehir MK, Logigian EL (2013) Electrodiagnosis of myotonic disorders. Phys Med Rehabil Clin N Am 24(1): 209-220.

16. Nagaraju K, Lundberg IE (2011) Polymyositis and dermatomyositis: pathophysiology. Rheum Dis Clin North Am 37(2): 159-171.

17. Mastaglia FL, Garlepp MJ, Phillips BA, Zilko PJ (2003) Inflammatory myopathies: clinical, diagnostic and therapeutic aspects. Muscle Nerve 27(4): 407-425.

18. Carrillo N, Malicdan MC, Huizing M (2018) GNE myopathy: etiology, diagnosis, and therapeutic challenges. Neurotherapeutics 15(4): 900914.

19. Milone M, Liewluck T (2019) The unfolding spectrum of inherited distal myopathies. Muscle Nerve 59(3): 283-294.
20. Basndwah A, Al-Mowalad E, Al-Said Y, Cupler E (2018) Hereditary Inclusion Body Myositis (IBM2) with a GNE c. 2228 T > C Mutation in 3 Saudi Arabian Families (P5.097)

21. Wenninger S, Schoser B (2018) Myasthenia gravis: current status of antibody diagnostics and aspects on refractory myasthenia gravis. Fortschr Neurol Psychiatr 86(9): 551-558.

22. Amir S, Qadir M, Usman M (2018) CLINICAL PROFILE OF PATIENTS WITH CARPAL TUNNEL SYNDROME. Khyber Medical University Journal $10(1)$.

23. Sonoo M, Menkes DL, Bland JD, Burke D (2018) Nerve conduction studies and EMG in carpal tunnel syndrome: do they add value?. Clin Neurophysiol Pract 3: 78-88.

24. Asheghan M, Hollisaz MT, Aghdam AS, Khatibiaghda A (2016) The prevalence of pronator teres among patients with carpal tunnel syndrome: Cross-sectional study. Int J Biomed Sci 12(3): 89-94.

25. Preston DC, Shapiro BE (2012) Electromyography and Neuromuscular Disorders E-Book: Clinical-Electrophysiologic Correlations (Expert Consult-Online and Print). Elsevier Health Sciences : 1.

26. Carter GT, Weiss MD, Friedman AS, Allan CH, Robinson L (2015) Diagnosis and treatment of work-related ulnar neuropathy at the elbow. Phys Med Rehabil Clin N Am 26(3): 513-522.

27. Mizer A, Bachmann A, Gibson J, Donaldson MB (2017) Self-report and subjective history in the diagnosis of painful neck conditions: A systematic review of diagnostic accuracy studies. Musculoskelet Sci Pract 31: 30-44.

28. Ferrante MA (2012) The thoracic outlet syndromes. Muscle Nerve 45(6): 780-795.

29. Dimberg EL (2012) Electrodiagnostic evaluation of ulnar neuropathy and other upper extremity mononeuropathies. Neurol Clin 30(2): 479503.

30. Hakimi K, Spanier D (2013) Electrodiagnosis of cervical radiculopathy. Phys Med Rehabil Clin N Am 24(1): 1-12.

31. Foley J, Finlayson H, Travlos A (2012) A review of thoracic outlet syndrome and the possible role of botulinum toxin in the treatment of this syndrome. Toxins (Basel) 4(11): 1223-1235.

32. Rhee JM, Yoon T, Riew KD (2007) Cervical radiculopathy. J Am Acad Orthop Surg 15(8): 486-494.

33. Laulan J, Fouquet B, Rodaix C, Jauffret P, Roquelaure Y, et al. (2011) Thoracic outlet syndrome: definition, aetiological factors, diagnosis, management and occupational impact. J Occup Rehabil 21(3): 366-373.

34. Latef TJ, Bilal M, Vetter M, Iwanaga J, Oskouian RJ, et al. (2018) Injury of the radial nerve in the arm: A review. Cureus. 10(2): e2199.

35. Shin AY, Spinner RJ, Steinmann SP, Bishop AT (2005) Adult traumatic brachial plexus injuries. J Am Acad Orthop Surg 13(6): 382-396.

36. Arnold WD, Krishna VR, Freimer M, Kissel JT, Elsheikh B (2012) Prognosis of acute compressive radial neuropathy. Muscle Nerve 45(6): 893-895.

37. Park HR, Lee GS, Kim IS, Chang JC (2017) Brachial plexus injury in adults. The Nerve 3(1): 1-1.

38. Edwards CC 2nd, Riew KD, Anderson PA, Hilibrand AS, Vaccaro AF (2003) Cervical myelopathy: current diagnostic and treatment strategies. Spine J 3(1): 68-81.

39. Sternberg ML, Gunter ML (2017) Syringomyelia. J Emerg Med 53(2): e31-e32.

40. Heiss JD, Jarvis K, Smith RK, Eskioglu E, Gierthmuehlen M, et al. (2019) Origin of syrinx fluid in syringomyelia: a physiological study. Neurosurgery 84(2): 457-468.

41. Reynolds J, Sachs G, Stavros K (2013) Chronic inflammatory demyelinating polyradiculoneuropathy (CIDP): clinical features, diagnosis, and current treatment strategies. R I Med J 99(12): 32-35.

42. Chhibber S, Greenberg SA (2011) Teaching Video NeuroImages: Widespread clinical myokymia in chronic inflammatory demyelinating polyradiculoneuropathy. Neurology 77(5): e33. 
43. Van den Bergh PY, Hadden RD, Bouche P, Cornblath DR, Hahn A, et al. (2010) European Federation of Neurological Societies/Peripheral Nerve Society guideline on management of chronic inflammatory demyelinating polyradiculoneuropathy: report of a joint task force of the European Federation of Neurological Societies and the Peripheral Nerve Society—first revision. Eur J Neurol 17(3): 356-363.

44. Breiner A, Brannagan TH $3^{\text {rd }}$ (2014) Comparison of sensitivity and specificity among 15 criteria for chronic inflammatory demyelinating polyneuropathy. Muscle nerve 50(1): 40-46.

45. Briani C, Brannagan III TH, Trojaborg W, Latov N (1996) Chronic inflammatory demyelinating polyneuropathy. Neuromuscul Disord 6(5): 311-325.

46. Magy L, Mathis S, Le Masson G, Goizet C, Tazir M, Vallat JM (2018) Updating the classification of inherited neuropathies: results of an international survey. Neurology 90(10): e870-e876.

47. Stojkovic T (2016) Hereditary neuropathies: an update. Rev Neurol (Paris) 172(12): 775-778.

48. Ramineni KK, Chandra SR, Mahadevan A, Kulkarni GB, Ramanujam CN (2019) Clinical, electrophysiological and laboratory parameters, and outcome in patients with biopsy proven systemic and nonsystemic vasculitic neuropathy. Neurol India 67(Supplement): S62-S70.

49. Rivière E, Cohen Aubart F, Maisonobe T, Maurier F, Richez C, et al. (2017) Clinicopathological features of multiple mononeuropathy associated with systemic lupus erythematosus: a multicenter study. J Neurol 264(6): 1218-1226

50. Enevoldson TP (2004) Recreational drugs and their neurological consequences. J Neurol Neurosurg Psychiatry 75(suppl 3): iii9-iii15.

51. Cottin V, Bel E, Bottero P, Dalhoff K, Humbert M, et al. (2017) Revisiting the systemic vasculitis in eosinophilic granulomatosis with polyangiitis (Churg-Strauss): a study of 157 patients by the Groupe d'Etudes et de Recherche sur les Maladies Orphelines Pulmonaires and the European Respiratory Society Taskforce on eosinophilic granulomatosis with polyangiitis (Churg-Strauss). Autoimmun Rev 16(1): 1-9.

52. Watson JC, Dyck PJ (2015) Peripheral neuropathy: a practical approach to diagnosis and symptom management. In Mayo Clin Proc 90(7): 940951.

53. Danjo J, Sawada H, Uchida K, Danjo S, Nakamura Y (2017) Efficacy of a new microvibration sensation measurement device at detecting diabetic peripheral neuropathy using a newly devised finger method. J Gen Fam Med 18(4): 155-161.

54. Rubin DI (2011) Acute and chronic polyradiculopathies. Continuum (Minneap Minn) 17(4): 831-854.

55. Bril V, Blanchette CM, Noone JM, Runken MC, Gelinas D, et al. (2016) The dilemma of diabetes in chronic inflammatory demyelinating polyneuropathy. J Diabetes Complications 30(7): 1401-1407. 